\author{
Anna Kurzaj \\ ORCID: 0000-0003-0586-5938 \\ Adam Mickiewicz Universität, Poznań \\ https://doi.org/10.19195/0435-5865.146.7
}

\title{
Zusammengesetzte Adjektive im deutsch-polnischen Vergleich anhand der Wörterbücher PWN
}

\begin{abstract}
s
In diesem Aufsatz werden die zusammengesetzten Adjektive im Deutschen und im Polnischen verglichen. In beiden Sprachen wurden sie bereits untersucht, jedoch wurden in jeder Sprache verschiedene Beschreibungsweisen eingesetzt. Das Ziel des Aufsatzes ist, die Unterschiede und Ähnlichkeiten in der Bildung von zusammengesetzten Adjektiven in beiden Sprachen zu untersuchen. Dargestellt wird eine quantitative und qualitative Analyse der möglichen Komposita anhand der untersuchten deutschpolnischen Wörterbücher. Es lässt sich schlussfolgern, dass es im Deutschen mehr Möglichkeiten gibt, neue zusammengesetzte Adjektive zu bilden. Im Polnischen lassen sich immerhin auch einige feste Wortbildungsmuster erkennen, die bei der Bildung von neuen Adjektiven angewendet werden können. Außerdem gibt es in beiden Sprachen Konstruktionen, die äquivalent oder ähnlich sind.
\end{abstract}

Schlüsselwörter: Wortbildung, Zusammensetzung, Adjektiv, kontrastive Grammatik, deutsch-polnischer Vergleich, Übersetzung

\section{Compound adjectives in German-Polish contrastive studies based on PWN dictionaries}

In this paper, compound adjectives in German and Polish are compared. In both languages they have already been examined. However, the grammars differ in the description method. The aim of this paper is to examine the differences and similarities in the forming of compound adjectives in both languages. The quantitative and qualitative analysis of possible compounds is carried out on the basis of German-Polish dictionaries. It can be concluded that in German there are more ways to form compound adjectives. Nevertheless, some word formation models can be identified in Polish, which can be used to form neologisms and hapax legomena. Furthermore, there are constructions in both languages that are equivalent or similar.

Keywords: word-formation, compound, adjective, contrastive grammar, German-Polish contrastive studies, translation 
Anna Kurzaj, Uniwersytet Adama Mickiewicza, Instytut Lingwistyki Stosowanej, Collegium Novum, Al. Niepodległości 4, 61-874 Poznań, E-Mail: annkur@amu.edu.pl.

Received: 17.04 .2020 , accepted: 28.02 .2021

Der deutsch-polnischen kontrastiven Grammatik wurden bereits viele ausführliche Bearbeitungen gewidmet, darunter ist vor allem die Arbeit von Ulrich Engel zu zählen (Engel 1999). Die Erkenntnisse dieser Lehre hatten einen unübersehbaren Einfluss auf die deutsch-polnische Translatorik und Sprachendidaktik. Mit dem Vergleich der grammatischen Konstruktionen können in beiden Sprachen Formen, die dieselben Funktionen erfüllen, bestimmt werden. Hierbei steht die Wortbildung beider Sprachen allerdings nicht im Fokus der kontrastiven Grammatik. In den deutschen Wortbildungsforschungsarbeiten wird betont, dass Zusammensetzungen eine der produktivsten Formen darstellen (vgl. Fleischer 1982: 15). Die Zusammensetzung ist ein Wort, das aus mindestens zwei selbständigen Morphemen besteht. Sie sind entweder gleichrangig (Kopulativkompositum), oder die zweite Komponente bildet das Grundwort, dessen Bedeutung von der ersten Komponente spezifiziert wird (Determinativkompositum) (Donalies 2007: 61). Häufig kommen in dieser Form Adjektive vor. Auch im Polnischen ist es möglich, zusammengesetzte Adjektive zu bilden. Die Produktivität der bestimmten Arten von zusammengesetzten Adjektiven kann sich jedoch zwischen den beiden Sprachen unterscheiden.

Das Ziel dieses Aufsatzes ist, die Möglichkeit, zusammengesetzte Adjektive zu bilden, im Deutschen und im Polnischen zu vergleichen. Bei dem Vergleich werden morphologische Bildungen und der Produktivitätsgrad von zusammengesetzten Adjektiven in beiden Sprachen nach ihrer morphologischen Bildung in Betracht gezogen. Die analysierten Adjektive stammen aus den deutsch-polnischen und polnischdeutschen Wörterbüchern PWN (Frączek / Wiktorowicz 2008a, 2008b). Es werden somit die Ähnlichkeiten und Unterschiede in der Wortbildung von zusammengesetzten Adjektiven beider Sprachen beschrieben. Da zusammengesetzte Adjektive im Deutschen viel häufiger vorkommen und mehr Wortbildungsmuster bilden als die im Polnischen, ist es dabei wichtig, auf mögliche Zusammensetzungen für die polnischen Adjektive hinzuweisen, die für die nur im Deutschen vorhandenen adjektivischen Wortbildungsmuster als Äquivalente gelten können. Obwohl im Vergleich nur auf Basis der Wörterbücher nicht alle zusammengesetzten Adjektive einer Sprache berücksichtigt werden, ermöglicht diese Analyse jedoch, das Vergleichsmaß in der Bildung zusammengesetzter Adjektive im Deutschen und Polnischen wahrzunehmen, da für die beiden Wörterbücher dieselben Herausgeber verantwortlich waren.

\section{Problem der Wortartbestimmung}

Die zusammengesetzten Adjektive im Deutschen sowie die möglichen Wortbildungsmuster der deutschen adjektivischen Zusammensetzungen wurden bereits ausführ- 
lich untersucht (vgl. Fleischer / Barz 2012: 320-331). Im Polnischen wurden ähnliche Recherchen durchgeführt, in denen die zusammengesetzten Adjektive analysiert wurden (vgl. Grzegorczykowa / Laskowski / Wróbel 1999: 510-520; Jadacka 2001: 105-109). Die Untersuchungen zu Wortbildung der Adjektive in diesen Sprachen unterscheiden sich jedoch in der morphologischen Beschreibungsweise. Besonders problematisch ist es in der Analyse der polnischen Adjektive, in welcher sich einige Ungenauigkeiten in der Bestimmung von Wortarten bemerken lassen. Selbst die Autoren deuten auf dieses Problem hin: Wörter wie wczesnośredniowieczny können entweder als Adjektiv-Nomen-Kompositum oder als Adverb-Adjektiv-Kompositum betrachtet werden (Grzegorczykowa / Laskowski / Wróbel 1999: 515). Die erste Interpretation ist jedoch etwas umstritten, da die Komponente -średniowieczny bereits ein Derivat beinhaltet, bei dem aus einem Nomen ein Adjektiv abgeleitet wird. In derselben Grammatik werden solche Wörter denominale Adjektive (przymiotniki odrzeczownikowe) genannt. Als Adjektive werden die entsprechenden Wörter auch im Deutschen betrachtet. Zu Adjektiven werden zudem die Komponenten gezählt, die mit anderen für Adjektive typischen Derivaten abgeleitet wurden.

Als problematisch gilt auch die Abgrenzung der Adjektive zu Adverbien, die als erste Komponente der polnischen Zusammensetzung fungieren. Das Problem ist auch am Beispiel von wczesnośredniowieczny zu bemerken. Aufgrund des Infixes -o- ist die erste Komponente entweder als Adverb oder Adjektiv zu interpretieren, d.h. die Zusammensetzung wurde entweder aus dem Adverb wcześnie oder Adjektiv wczesny gebildet. In der deutschen Entsprechung frühmittelalterlich gilt die Komponente früh als Adjektiv, weil sie das Hauptkriterium von Adjektiven erfüllt:

Wörter, die in der Klammer zwischen Determinativ und Nomen auftreten können und in der Positionierung in Genus, Numerus und Kasus vom Nomen bestimmt werden und/oder prädikativ außerhalb eines Verbalkomplexes kopularegiert auftreten und in der Positionierung indeklinabel bleiben. (Trost 2006: 2)

An dieser Stelle muss bemerkt werden, dass viele solcher Beispiele ihre direkten Entsprechungen in beiden Sprachen aufweisen, deshalb ist es sinnvoll, sie als gleichwertig zu betrachten. So werden die ersten Komponenten, die im Polnischen entweder als Adverbien oder als Adjektive betrachtet werden können, zum Zweck der Analyse als Adjektive interpretiert.

\section{Haupteinteilung - quantitative Analyse}

Zusammengesetzte Adjektive können im Deutschen folgende Wortbildungskonstruktionen bilden: Adjektiv-Adjektiv; Adverb-Adjektiv; Nomen-Adjektiv; Zahlwort-Adjektiv; Präposition-Adjektiv; Pronomen-Adjektiv; Verb-Adjektiv.

Das Polnische bietet hingegen Zusammensetzungen aus Verb und Adjektiv nicht an. Die anderen aufgeführten Wortbildungskonstruktionen sind im Polnischen jedoch vorhanden. Der quantitative Vergleich stellt die untere Tabelle dar. 
Tab. 1: Quantitativer Vergleich von Zusammensetzungsarten im Deutschen und Polnischen ${ }^{1}$

\begin{tabular}{|l|c|c|}
\hline $\begin{array}{c}\text { Art der Zusammenset- } \\
\text { zung }\end{array}$ & $\begin{array}{c}\text { Anzahl (Prozentanteil) } \\
\text { Deutsch }\end{array}$ & $\begin{array}{c}\text { Anzahl (Prozentanteil) } \\
\text { Polnisch }\end{array}$ \\
\hline Adjektiv-Adjektiv & $318(18,25 \%)$ & $175(23,58 \%)$ \\
\hline Adverb-Adjektiv & $32(1,83 \%)$ & $2(0,27 \%)$ \\
\hline Nomen-Adjektiv & $1049(60,22 \%)$ & $58(7,82 \%)$ \\
\hline Zahlwort-Adjektiv & $173(9,93 \%)$ & $246(33,15 \%)$ \\
\hline Präposition-Adjektiv & $29(1,66 \%)$ & $250(33,69 \%)$ \\
\hline Pronomen-Adjektiv & $27(1,55 \%)$ & $11(1,48 \%)$ \\
\hline Verb-Adjektiv & $114(6,54 \%)$ & $0(0 \%)$ \\
\hline \multicolumn{1}{|c|}{ Summe } & $\mathbf{1 7 4 2}$ & $\mathbf{7 4 2}$ \\
\hline
\end{tabular}

Da die zusammengesetzten Adjektive im Wörterbuch Deutsch-Polnisch eine größere Gruppe der Lemmata darstellen als im Wörterbuch Polnisch-Deutsch, wird nachgewiesen, dass sie im Deutschen häufiger auftreten und somit als produktiver gelten. Auch im Prozentanteil der bestimmten Arten von Zusammensetzungen sind Unterschiede in der Wortbildung von Adjektiven in beiden Sprachen ersichtlich. Im Wörterbuch Deutsch-Polnisch bildet das Muster „Nomen-Adjektiv“ die größte Gruppe der zusammengesetzten Adjektive. Folgend kommen zudem „Adjektiv-Adjektiv“"Zusammensetzungen häufig vor. Sie treten in dem Wörterbuch allerdings viel seltener als Nomen-Adjektiv-Komposita auf. Die Aufmerksamkeit sollte auch auf Zahlwort-Adjektiv- und Verb-Adjektiv-Komposita gelenkt werden. Andere Arten der adjektivischen Zusammensetzungen spielen laut Prozentanteil eine marginale Rolle. Die im Wörterbuch Polnisch-Deutsch am häufigsten vorkommenden Zusammensetzungsarten sind dagegen „Präposition-Adjektiv“ und „Zahlwort-Adjektiv“. Eine andere wesentliche Art der Zusammensetzung stellen im Polnischen Adjektiv-Adjektiv-Komposita dar: Ihr Prozentanteil beträgt ungefähr 24\%. Nomen-Adjektiv-Komposita treten dagegen im Polnischen viel seltener auf und ihr Prozentanteil beläuft sich auf 7,82\%. Andere Arten von zusammengesetzten Adjektiven (d.h. Pronomen-Adjektiv- und Adverb-Adjektiv-Komposita) kommen äußerst selten vor und können als nicht produktiv gelten.

\section{Weitere Materialeinteilung - quantitative und qualitative Analyse}

Die zusammengesetzten Adjektive lassen sich noch genauer einteilen, wenn spezifiziert wird, aus welcher Wortart ein Adjektiv abgeleitet wurde. In der Gruppe von Adjektiv-Adjektiv-Komposita werden folgende Untergruppen unterschieden:

\footnotetext{
${ }^{1}$ Alle Tabellen wurden von der Autorin des Aufsatzes anhand der Analyse erstellt.
} 
Tab. 2: Typen der Zusammensetzung aus zwei Adjektiven

\begin{tabular}{|l|l|c|c|}
\hline \multicolumn{1}{|c|}{ Art der Zusammensetzung } & \multicolumn{1}{c|}{ Abkürzung } & Deutsch & Polnisch \\
\hline Adjektiv (Nomen)+Adjektiv & A (N)+A & 3 & 6 \\
\hline Adjektiv (Nomen)+Adjektiv (Nomen) & A (N)+A (N) & 2 & 14 \\
\hline Adjektiv (Nomen)+Adjektiv (Verb) & A (N)+A (V) & 0 & 1 \\
\hline Adjektiv (Verb)+ Adjektiv (Verb) & A (V)+A (V) & 1 & 0 \\
\hline Adjektiv+Adjektiv & A+A & 100 & 20 \\
\hline Adjektiv+Adjektiv (Nomen) & A+A (N) & 151 & 115 \\
\hline Adjektiv+Adjektiv (Verb) & A+A (V) & 54 & 19 \\
\hline Adjektiv+Adjektiv+Adjektiv & A+A+A & 4 & 0 \\
\hline Adjektiv+Adjektiv+Adjektiv+Adjektiv & A+A+A+A & 2 & 0 \\
\hline Adjektiv+Nomen+Adjektiv & A+N+A & 1 & 0 \\
\hline
\end{tabular}

Der Tabelle können einige Ähnlichkeiten zwischen beiden Sprachen entnommen werden: Die größte Untergruppe stellen in beiden Sprachen „Komposita des Typs“ $\mathrm{A}+\mathrm{A}(\mathrm{N})$ dar; auch $\mathrm{A}+\mathrm{A}$ und $\mathrm{A}+\mathrm{A}(\mathrm{V})$ sind in ähnlichem Maße präsent. Die Untergruppen wie $A(N)+A$ und $A(N)+A(V)$ kommen nur gelegentlich vor. Der größte Unterschied ist bei $A(N)+A(N)$ zu sehen: Auf der polnischen Seite wird diese Gruppe von mehreren Beispielen repräsentiert.

Komposita des Typs „Adjektiv (Nomen)+Adjektiv“ stellen im Deutschen vor allem Kopulativkomposita dar, beispielsweise sportlich-elegant. Im Polnischen stellen Komposita des Typs A (N)+A dagegen Farbwörter dar, bei welchen das nominale Adjektiv das Hauptwort spezifiziert: krwawoczerwony, krwistoczerwony, ognistoczerwony, mlecznobiały, śnieżnobiały, stalowoszary.

Für den Typ A (N)+A (N) sind im Deutschen ebenso Kopulativkomposita charakteristisch (manisch-depressiv; kaiserlich-königlich). Im Polnischen ist dieser Typ für Adjektive typisch, die Himmelsrichtungen (pótnocno-wschodni, potudniowo-zachodni) und Regionen (zachodnioeuropejski, poludniowoameryński, środkowoeuropejski) bezeichnen.

Der Typ A $(\mathrm{N})+\mathrm{A}(\mathrm{V})$ ist dagegen sowohl für die deutsche als auch für die polnische Sprache nicht typisch. Im Deutschen ist er nicht vorhanden und im Polnischen wurde nur ein Beispiel gefunden - narodowowyzwoleńczy.

Noch seltener repräsentiert in beider Sprachen ist der Typ „Adjektiv (Verb)+Adjektiv (Verb)": Im deutschen Wörterbuch ist berühmt-berüchtigt das einzige Beispiel, das ein Kopulativkompositum darstellt und aus Partizipien gebildet wird. Nach diesem Muster können auch andere Zusammensetzungen von diesem Typ gebildet werden, die nicht lexikalisiert sind. Auch auf der polnischen Seite ist die Bildung von analogen Kopulativkomposita möglich. Da Kopulativkomposita in der Regel nicht lexikalisiert sind und häufig Ad-hoc-Bildungen darstellen, sind sie im Wörterbuch nicht vorhanden. 
Für den Zusammensetzungstyp, in dem ein Wort aus zwei unabgeleiteten Adjektiven stammt, sind im Deutschen Farbwörter charakteristisch, bei welchen beide Komponenten Farben benennen, z.B. blaugrün, grauschwarz, oder die erste Komponente auf eine Abtönung hinweist, z.B. tiefschwarz, zartrosa, dunkelgrün, hellblau (Trost 2006: 115). In dieser Gruppe kommen sowohl Kopulativ- (süßsauer, taubstumm) als auch Determinativkomposita (mittelgro $\beta$, hochmodern) vor, was darauf hinweist, dass dieser Typ produktiv ist. Im Wörterbuch Polnisch-Deutsch wird diese Gruppe vor allem anhand von analogen Farbwörtern, wie ciemnoniebieski, jasnozielony oder żóltozielony repräsentiert. Eine weitere Ähnlichkeit mit der deutschen Gruppe lässt sich in der Bildung von sowohl Kopulativ- (gluchoniemy), als auch Determinativkomposita (petnotlusty, starodawny) finden. Es gibt daher keine qualitativen Unterschiede bei diesem Typ zwischen dem Deutschen und Polnischen.

Beim Typ A+A (N) wurde die zweite Komponente von einem Nomen abgeleitet. In beiden Sprachen kommt dieser Typ unter Adjektiv-Adjektiv-Komposita am häufigsten vor. Auf der deutschen Seite lässt sich erkennen, dass die zweiten Komponenten vor allem mit dem Suffix -ig abgeleitet werden und sich häufig auf Körperteile beziehen: weichherzig, scharfzüngig, leichtfüßig, langhaarig, auch wenn die gesamte Zusammensetzung eine übertragene Bedeutung trägt. Im Polnischen beziehen sich viele Zusammensetzungen von diesem Typ ebenfalls auf Körperbezeichnungen: błękitnooki, dlugonogi, ciemnoskóry. In diesem Fall werden im Polnischen paradigmatische Suffixe $-i$ oder $-y$ angewendet. Bemerkenswert ist hierbei, dass diese Zusammensetzungen sich im Polnischen auf das Äußere beziehen, während im Deutschen dieser Typ auch in Bezug auf die inneren Organe gebildet wird, z.B. leichtherzig. Sowohl im Deutschen als auch im Polnischen werden mit diesem Typ vor allem Determinativkomposita gebildet: dreigeistig, równoboczny usw.

Ein weiteres produktives Modell in beiden Sprachen stellt der Typ A+A (V) dar. Dabei ist zu bemerken, dass die zweiten Komponenten der deutschen Zusammensetzungen am häufigsten Partizipien sind: hochfliegend, schwerwiegend, neuvermählt, hocherfreut. Da Partizipien im Deutschen auf eine regelmäßige Weise gebildet werden, lässt sich vermuten, dass sie für die Neubildungen gebräuchlich sind. Die Zusammensetzungen mit Partizipien als zweite Komponente kommen ebenso im Polnischen vor, wie długograjacy, ogólnoksztatcacy, und sie stellen zugleich ein produktives Modell dar. Die zweiten Komponenten können in der polnischen Sprache auch häufig von Verben mit einem Suffix abgeleitet werden, z.B. -ny: pierworodny, lekkostrawny, szybkostrzelny. Auf der deutschen Seite wird an dieser Stelle das produktive Suffix -ig gebraucht: kurzlebig, vollzählig.

Die längeren Zusammensetzungen, die aus mehr als zwei Komponenten gebildet wurden, sind nur im Wörterbuch Deutsch-Polnisch präsent. Solche Wörter bezeichnen die genauere Spezifizierung einer Eigenschaft: spätmittelhochdeutsch. Im Polnischen ist die Bildung von analogen Zusammensetzungen theoretisch möglich (późnośredniowysokoniemiecki), jedoch kommen sie deutlich seltener vor und haben einen fachwissenschaftlichen Charakter. 
Die nächste Gruppe stellen Komposita aus Adverb und Adjektiv dar. In beiden Wörterbüchern werden diese nur mit wenigen Beispielen repräsentiert. Die beispielsstärkste Untergruppe im Deutschen ist $\mathrm{Adv}+\mathrm{A}(\mathrm{V})$. Dabei ist es der einzige Typ der Adverb-Adjektiv-Komposita, der im Wörterbuch Polnisch-Deutsch vorhanden ist.

Tab. 3: Typen der Zusammensetzung aus Adverb und Adjektiv

\begin{tabular}{|l|l|c|c|}
\hline \multicolumn{1}{|c|}{ Art der Zusammensetzung } & Abkürzung & Deutsch & Polnisch \\
\hline Adverb+Adjektiv & $\mathrm{Adv}+\mathrm{A}$ & 8 & 0 \\
\hline Adverb+ Adjektiv (Nomen) & $\mathrm{Adv}+\mathrm{A}(\mathrm{N})$ & 8 & 0 \\
\hline Adverb+ Adjektiv (Verb) & $\mathrm{Adv}+\mathrm{A}(\mathrm{V})$ & 16 & 2 \\
\hline
\end{tabular}

Für all diese Typen sind in deutschen Zusammensetzungen Beispiele zu finden, die mithilfe des Adverbs wohl gebildet wurden: wohlfeil, wohlklingend, wohlbedacht, wohltätig. Sie bezeichnen vor allem eine intensivierte Eigenschaft, gegebenenfalls beziehen sie sich auf ihre gute Qualität. Mit wohl könnten auch andere nicht lexikalisierte Zusammensetzungen gebildet werden. Zudem sind im Wörterbuch Deutsch-Polnisch auch Beispiele zu finden, die idiomatisiert und daher unproduktiv sind, z.B. immergrün, nimmersatt.

Die einzigen Beispiele für das Polnische sind lewoskrętny und prawoskrętny, die entsprechend als ,skręcający w lewo' (nach links abbiegend) und ,skręcający w prawo' (nach rechts abbiegend) erläutert werden können. Weil es keine anderen Richtungen gibt, in die man abbiegen könnte, ist dieses Muster nicht produktiv und andere Beispiele dieses Typs können im Polnischen nicht gebildet werden.

Die folgende Gruppe der adjektivischen Zusammensetzungen stellen NomenAdjektiv-Komposita dar. Wie in der Haupteinteilung beschrieben, bilden sie die größte Gruppe der deutschen zusammengesetzten Adjektive, während sie im Polnischen keine wesentliche Gruppe darstellen. Im Deutschen sind ebenfalls mehrere Typen von Nomen-Adjektiv-Komposita vorhanden. Wichtig zu bemerken ist, dass die häufigsten Typen im Deutschen [N+A, N+A (N); N+A(V)] auch im Polnischen vorkommen. Zunächst werden Typen besprochen, die in beiden Sprachen vorkommen.

Tab. 4: Typen der Zusammensetzung aus Nomen und Adjektiv

\begin{tabular}{|l|l|c|c|}
\hline \multicolumn{1}{|c|}{ Art der Zusammensetzung } & \multicolumn{1}{c|}{ Abkürzung } & Deutsch & Polnisch \\
\hline Nomen (Adjektiv)+Adjektiv & $\mathrm{N}(\mathrm{A})+\mathrm{A}$ & 4 & 0 \\
\hline Nomen+Adjektiv & $\mathrm{N}+\mathrm{A}$ & 694 & 11 \\
\hline Nomen+ Adjektiv (Nomen) & $\mathrm{N}+\mathrm{A}(\mathrm{N})$ & 138 & 9 \\
\hline Nomen+ Adjektiv (Präposition) & $\mathrm{N}+\mathrm{A}($ Prä) & 12 & 0 \\
\hline Nomen+ Adjektiv (Verb) & $\mathrm{N}+\mathrm{A}(\mathrm{V})$ & 191 & 38 \\
\hline Nomen+Nomen+Adjektiv & $\mathrm{N}+\mathrm{N}+\mathrm{A}$ & 9 & 0 \\
\hline Nomen+Nomen+ Nomen+Adjektiv & $\mathrm{N}+\mathrm{N}+\mathrm{N}+\mathrm{A}$ & 1 & 0 \\
\hline
\end{tabular}


Der Typ N+A tritt im Wörterbuch Deutsch-Polnisch am häufigsten auf. Durch Nomen wird die Bedeutung des Adjektivs spezifiziert bzw. intensiviert, also sind diese Bildungen Determinativkomposita: bildschön, eiskalt, glasklar, aidskrank, honigsüß . Es kann sogar festgestellt werden, dass der quantitative Unterschied zwischen deutschen und polnischen Zusammensetzungen vor allem auf diese Gruppe zurückzuführen ist. Im Deutschen lassen sich hier etliche feste Muster bemerken, z.B.: -arm (kalorienarm), -bereit (dienstbereit), -frei (fieberfrei), -schwach (leistungsschwach), -sicher (einbruchssicher), -reif(spruchreif). Typisch sind in dieser Gruppe auch Farbwörter wie: apfelgrün, kaffeebraun, weingelb. Solche Farbwörter könnten jedoch durch den Typ A (N)+A wiedergegeben werden.

Auch im Polnischen lassen sich in dieser Gruppe einige Wortbildungsmuster erkennen: -trwaty (ogniotrwaty), -szczelny (dźwiękoszczelny), -odporny (kuloodporny), -podobny (skóropodobny), -czuły (świattoczuły). Mithilfe dieser Muster können auch andere zusammengesetzte Adjektive gebildet werden, die Okkasionalismen darstellen, z.B. granatoodporny, wannopodobny. Bei dem Vergleich dieser Zusammensetzungsart muss bemerkt werden, dass die polnischen Adjektive viel seltener Simplizia darstellen. Deutlich häufiger werden sie von anderen Wortarten abgeleitet. Deshalb könnten die direkten Entsprechungen von deutschen Zusammensetzungen $\mathrm{N}+\mathrm{A}$ zu anderen Typen gezählt werden.

Zum Typ $\mathrm{N}+\mathrm{A}(\mathrm{N})$ zählt man im Deutschen ebenfalls einige feste Muster. Sehr produktiv ist beispielsweise -förmig als zweite Komponente: eiförmig, $h a-$ kenförmig. Auch -pflichtig und -süchtig kommen häufig vor: steuerpflichtig, streitsüchtig. Unter Umständen sind hier auch Muster wie -freudig, -farbig und -würdig zu zählen. Die zweiten Komponenten wurden von Nomina vor allem mit dem produktiven Suffix -ig abgeleitet. Im Wörterbuch Deutsch-Polnisch sind fünf Beispiele zu finden, die sich auf geographische Bezeichnungen beziehen, z.B.: südeuropäisch, angelsächsisch. Ähnliche Bildungen kommen auch im Wörterbuch Polnisch-Deutsch vor. Die Erstglieder werden dabei häufig abgekürzt: anglosaski, iberoamerykański. Viel häufiger werden allerdings die Komposita, die sich auf Regionen beziehen, zum Typ A (N)+A (N) gezählt, z.B. poludniowoeuropejski. Außerdem ist in diesem Typ das Muster -języczny zu erkennen, das als produktiv anzusehen ist. Das folgende Beispiel bezieht sich auf einen Körperteil, nämlich jajoglowy. Jedoch muss erwähnt werden, dass das Wort eine metaphorische Bedeutung trägt und kein festes Modelldarstellt im Gegensatz zu den bereits beschriebenen Mustern -wlosy oder -oki.

Der Typ N+A (V) tritt dagegen sowohl im Deutschen als auch im Polnischen auf. Im Deutschen kommen verbale Adjektive vor allem als Partizipien vor: Partizip I (44 Beispiele, z.B.: energiesparend, gewinnbringend) und Partizip II (56 Beispiele, z.B. fachbezogen, preisgekrönt). Zudem sind einige feste Muster auf der deutschen Seite zu bemerken: -abhängig (heroinabhängig), -schädlich (umweltschädlich), -durchlässig (luftdurchlässig), -bedürftig (pflegebedürfig), -empfindlich (kälteempfindlich). Viele feste Muster sind in diesem Typ ebenso im Pol- 
nischen zu finden: -naśladowczy (dźwiękonaśladowczy), -bójczy (bakteriobójczy), -leczniczy (wodoleczniczy), -twórczy (chorobotwórczy), -chłonny (energochłonny), -oszczędny (materiatooszczędny), -nośny (krwionośny), -żerny (rożlinożerny), -dajny (miarodajny), -lubny (cieptolubny). Einige von ihnen werden als produktiv angesehen: Sie bilden Zusammensetzungen, die im Wörterbuch Polnisch-Deutsch nicht gefunden werden können, z.B.: zimnolubny, wirusobójczy, wododajny. Der größte Unterschied zwischen den deutschen und polnischen Zusammensetzungen des Typs $\mathrm{N}+\mathrm{A}(\mathrm{V})$ besteht darin, dass die Grundwörter der polnischen Beispiele nicht als Partizipien auftreten, sondern die Adjektive ausschließlich mit verschiedenen Suffixen $(-c z y,-n y)$ abgeleitet werden.

Die erste Komponente wird im Typ N (A)+A von einem Nomen abgeleitet und bezieht sich auf Personengruppen: totenblass, totenbleich, totenstill und behindertengerecht. Im Polnischen kommt dieser Wortbildungstyp nicht vor.

Einen eher ungewöhnlichen Typ bilden Zusammensetzungen N+A (Prä), die nur im Deutschen vorkommen und selbst in dieser Sprache nicht zahlreich repräsentiert werden. Ein festes Muster stellt hier -widrig dar, bei welchem das Adjektiv von der Präposition, wider' mit dem Suffix -ig abgeleitet wurde: gesetzwidrig, ordnungswidrig, sittenwidrig. Ein anderes Beispiel ist eidesstattlich, das eher einen Sonderfall in der Bildung von Zusammensetzungen darstellt.

Im Deutschen werden auch Zusammensetzungen der Gruppe Nomen+Adjektiv aus mehreren Komponenten gebildet, zudem treten sie als intensivierende Determinativkomposita auf: funkelnagelneu, kohlpechrabenschwarz. Die entsprechenden Bildungen sind im Polnischen nicht möglich.

Eine weitere bedeutende Gruppe von adjektivischen Zusammensetzungen stellen Komposita aus Zahlwort und Adjektiv dar. Prozentual gesehen sind solche Bildungen im Polnischen viel produktiver als im Deutschen. Wird jedoch die Anzahl der in den Wörterbüchern gefundenen Beispiele betrachtet, ist der Unterschied nicht sehr groß. Dabei kommen in beiden Sprachen Verbindungstypen vor, die in der anderen Sprache nicht vorhanden sind.

Wie in der Tabelle dargestellt, verbinden sich Adjektive mit verschiedenen Arten von Zahlwörtern, indem sie Zusammensetzungen bilden. Es steht jedoch auBer Zweifel, dass die beispielsstärkste Gruppe darunter der Typ ZK+A (N) ist. Sowohl im Wörterbuch Deutsch-Polnisch, als auch im Wörterbuch Polnisch-Deutsch kommt diese Gruppe am häufigsten vor. Auch der Typ ZUn+A (N) ist unter den Zusammensetzungen aus Zahlwörtern und Adjektiven von großer Bedeutung. Der auffälligste quantitative Unterschied ist in dem Typ ZKol+A (N) zu sehen. 
Tab. 5: Typen der Zusammensetzung aus Zahlwort und Adjektiv

\begin{tabular}{|l|l|c|c|}
\hline \multicolumn{1}{|c|}{ Art der Zusammensetzung } & \multicolumn{1}{c|}{ Abkürzung } & Deutsch & Polnisch \\
\hline Kardinalzahlwort+Adjektiv (Nomen) & ZK+A (N) & 114 & 139 \\
\hline Kardinalzahlwort+Adjektiv (Verb) & ZK+A (V) & 1 & 2 \\
\hline Kollektivzahlwort+Adjektiv (Nomen) & ZKol+A (N) & 2 & 15 \\
\hline Kollektivzahlwort+Adjektiv (Verb) & ZKol+A (V) & 0 & 1 \\
\hline Ordinalzahlwort+Adjektiv & ZO+A & 1 & 0 \\
\hline Ordinalzahlwort+Adjektiv (Nomen) & ZO+A (N) & 4 & 8 \\
\hline Ordinalzahlwort+Adjektiv (Verb) & ZO+A (V) & 2 & 0 \\
\hline unbestimmtes Zahlwort+Adjektiv (Verb) & ZUn+A (V) & 2 & 0 \\
\hline unbestimmtes Zahlwort+Adjektiv (Nomen) & ZUn+A (N) & 21 & 57 \\
\hline Wiederholungszahlwort+Adjektiv & ZW+A & 1 & 0 \\
\hline Zahlbruchwort+Adjektiv & ZB+A & 15 & 15 \\
\hline Zahlbruchwort+Adjektiv (Nomen) & ZB+A (N) & 9 & 8 \\
\hline Zahlbruchwort+Adjektiv (Verb) & ZB+A (V) & 0 & 1 \\
\hline Zahlbruchwort+Adjektiv+Adverb & ZB+A+Adv & 1 & 0 \\
\hline
\end{tabular}

Im Falle der Zahlwörter verbinden sich die Adjektive vor allem mit Kardinalia. Nominale Adjektive formen solche feste Muster wie: -jährig, -monatig oder -stündig im Deutschen und -letni, -miesięczny, -godzinny dementsprechend im Polnischen. Diese Muster beziehen sich auf Zeitbezeichnungen. Darüber hinaus gibt es im Deutschen weitere Muster, in denen Adjektive von Nomina mit dem Suffix -ig abgeleitet werden, z.B.: achtkantig, sechszeilig usw. Auf der polnischen Seite werden Adjektive vor allem mit dem Suffix -owy abgeleitet: czteroosobowy, pięciopokojowy oder mit dem Suffix -ny: sześciokatny. Semantisch gesehen gibt es in diesem Typ keine Unterschiede zwischen beiden Sprachen.

Viele Ähnlichkeiten lassen sich zudem im Typ ZK+A (V) erkennen: Dieser Typ gilt in beiden Sprachen als unproduktiv und seine Beispiele stellen semantische Entsprechungen dar: zweideutig - dwuznaczny. Dabei kann die zweite Komponente im Polnischen als Partizip fungieren: jednobrzmiacy. Eine dementsprechende Form kommt im Deutschen nicht vor.

Wie bereits in der Tabelle 5 aufgeführt, ist im Typ ZKol+A (N) ein bedeutender quantitativer Unterschied zu finden. Im Deutschen tritt die erste Komponente nur als Zahlwort „beide“ auf: beid(er)seitig. Es scheint, dass der Typ im Polnischen komplexer aufgebaut ist: Neben den direkten Entsprechungen der Komposita mit beid- (auf Polnisch: obu-: oburęczny, obopólny) sind hier auch Beispiele mit trójoder czworo- zu sehen: trójbarwny, trójstronny, czworonożny. Bemerkenswert ist jedoch, dass die direkten zusammengesetzten Entsprechungen der angeführten Beispiele auch im Deutschen möglich sind (vierbeinig, dreifarbig, dreiseitig). Sie werden allerdings zum Typ ZK+A (N) gezählt. Der Typ ZKol+A (V) ist dagegen 
nur auf der polnischen Seite präsent (trójdzielny). Obwohl die gleiche Bildung auch im Deutschen möglich ist, wurde sie im Wörterbuch Deutsch-Polnisch nicht berücksichtigt.

Eine weitere bedeutende Gruppe von Zahlwort-Adjektiv-Zusammensetzungen bilden Komposita mit Ordinalia: $\mathrm{ZO}+\mathrm{A}, \mathrm{ZO}+\mathrm{A}(\mathrm{N}), \mathrm{ZO}+\mathrm{A}(\mathrm{V})$. Ersteres ist nur im Deutschen möglich. Ein Beispiel dafür stellt die Zusammensetzung drittletzt dar. Analoge Bildungen können mit anderen Ordinalzahlwörtern und dem Adjektiv letzt gebildet werden. Die direkte Entsprechung im Polnischen ist keine Zusammensetzung, sondern formt eine Phrase: trzeci od końca. Auch der Typ ZO+A (V) kommt laut des Ergebnisses der Analyse nur im Deutschen vor, z.B. erstgeboren, erstgenannt. Unter Umständen kann das Wort pierworodny auf der polnischen Seite zu diesem Typ gezählt werden. Ansonsten werden die polnischen Entsprechungen von solchen Komposita eher als Phrasen wiedergegeben: wspomniany jako pierwszy. Im Typ ZO+A (N) sind dagegen viele Ähnlichkeiten zwischen beiden Sprachen ersichtlich. Auf der deutschen Seite können solche Beispiele wie: erstklassig, drittklassig, erstrangig gefunden werden, während es auf der polnischen „Seite“ Wörter gibt wie pierwszoligowy, trzecioligowy, pierwszorzędny usw., die die direkten Entsprechungen der deutschen Beispiele sind. Darüber hinaus sind im Polnischen Zusammensetzungen dieses Typs aus Datumsbezeichnungen möglich, z.B. pierwszomajowy, die im Deutschen nicht vorkommen.

Sowohl im Deutschen als auch im Polnischen können die adjektivischen $\mathrm{Zu}-$ sammensetzungen mit unbestimmten Zahlwörtern gebildet werden. Dabei kann die zweite Komponente im Deutschen aus einem Verb abgeleitet sein, dies entspricht dem Typ ZUn+A (V): vielsagend, vieldeutig. Eine eventuell analoge Bildung des Typs ZUn+A (V) ist auch im Polnischen möglich: wieloznaczny - die direkte Entsprechung von vieldeutig. Am häufigsten kommen unter Komposita mit unbestimmten Zahlwörtern Zusammensetzungen des Typs $\mathrm{ZUn}+\mathrm{A}(\mathrm{N})$ vor. Im Deutschen werden diese Komposita insbesondere mit viel und mehr; und aus den mit dem Suffix -ig abgeleiteten Adjektiven gebildet: mehrbändig, mehrstimmig, vielsprachig, vielseitig. Im Polnischen sind analoge Bildungen zu sehen: wielogłosowy, kilkustonny, kilkupiętrowy. Dabei werden die Adjektive am häufigsten mit dem Suffix -owy gebildet. Allerdings ist dieses Suffix nicht immer anwendbar: Gelegentlich wird das Adjektiv mit dem Suffix -ny abgeleitet: wieloramienny, wielodzietny. So gilt das polnische -owy nicht immer als Entsprechung für das deutsche -ig.

Eine weitere Untergruppe von Zahlwort-Adjektiv-Zusammensetzungen stellen in beiden Sprachen Komposita mit Bruchzahlwörtern als erste Komponente dar. Sowohl im Deutschen als auch im Polnischen treten folgende Typen auf: $\mathrm{ZB}+\mathrm{A} ; \mathrm{ZB}+\mathrm{A}(\mathrm{N})$. Hierbei kommen die ersten Komponenten vor allem als Bruchzahlwörter halb- und pót- vor, die als direkte Entsprechungen in beiden Sprachen gelten: Im Typ ZB+A sind das: halbnackt - pótnagi; halboffen - pótotwarty. Im Typ ZB+A (N): halbjährig - pótroczny, halbstündig - pótgodzinny. Obwohl der 
Typ ZB+A (V) auf Basis des Vergleichs nur im Polnischen vorkommt (półleżacy), ist die analoge Bildung ebenfalls im Deutschen möglich und produktiv, z.B. halbliegend. Ähnliche Bildungen können zudem mit Wörtern wie anderthalb - póttora als erste Komponente geformt werden: anderthalbstündig - póltoragodzinny.

Die Typen, die nur auf der deutschen Seite auftreten, sind ZW+A und $\mathrm{ZB}+\mathrm{A}+\mathrm{Adv}$. Bemerkenswert ist jedoch, dass der ersterwähnte Typ nur durch ein Beispiel vertreten ist, das eine lexikalisierte, unproduktive Bildung darstellt: neunmalklug. Die Zusammensetzung des Typs $\mathrm{ZB}+\mathrm{A}+\mathrm{Adv}$ ist auch unproduktiv und lediglich am Beispiel halbimmergrün zu sehen.

Eine wesentliche Gruppe seitens der polnischen Sprache stellen Zusammensetzungen aus Präposition und Adjektiv dar. Obwohl solche Bildungen auch im Deutschen möglich sind, werden sie im Wörterbuch Deutsch-Polnisch weniger zahlreich repräsentiert.

Tab. 6: Typen der Zusammensetzung aus Präposition und Adjektiv

\begin{tabular}{|l|l|c|c|}
\hline \multicolumn{1}{|c|}{ Art der Zusammensetzung } & Abkürzung & Deutsch & Polnisch \\
\hline Präposition+Adjektiv & Prä+A & 8 & 18 \\
\hline Präposition+Adjektiv (Nomen) & Prä+A (N) & 18 & 230 \\
\hline Präposition+Adjektiv (Verb) & Prä+A (V) & 3 & 2 \\
\hline
\end{tabular}

Der beispielsstärkste Typ der deutschen sowie polnischen Zusammensetzungen ist Prä $+\mathrm{A}(\mathrm{N})$, im Polnischen wird der Typ jedoch mit mehreren Beispielen belegt. Viel seltener treten die Präposition-Adjektiv-Zusammensetzungen als Typ Prä+A auf. Der Typ Prä+A (V) lässt sich hinsichtlich ihrer Anzahl als unproduktiv bezeichnen.

Beim Typ Prä+A (N) sind die ersten Komponenten im Deutschen solche Präpositionen wie: außer (außerbetrieblich, außergerichtlich), zwischen (zwischenmenschlich, zwischenzeitlich) oder wider (widersinnig, widerrechtlich). In der polnischen Sprache können verschiedene Präpositionen als erste Komponente auftreten: $c^{-}{ }^{2}$ (cogodzinny), do- (dożylny), między- (międzygwiezdny), nad- (nadbrzeżny), pod- (podskórny), przed- (przedślubny), przeciw- (przeciwbakteryjny) usw. Auch Komposita mit bez-, deren deutschen Entsprechungen als Ableitungen gelten (bezsensowny - sinnlos) dürfen zu diesem Typ gezählt werden. Der Typ Prä+A kann dagegen im Deutschen mit der Präposition vor- (vorlaut, vorletzt) gebildet werden, während im Polnischen dieser Zusammensetzungstyp vor allem mit bez gebildet wird: bezsilny, bezwolny. Verbindungen mit anderen Präpositionen sind in diesem Typ auch möglich, z.B.: przedostatni. Der Typ Prä+A(V)

2 Dabei muss darauf aufmerksam gemacht werden, dass co je nach Satzfunktion zu verschiedenen Wortarten gezählt wird (z.B. Frageadverbien, Pronomina, Partikeln). Wenn es auf die Frequenz einer Tätigkeit hinweist und ein Nomen im Akkusativ fordert (z.B. co godzinę, co tydzień), wird das Wort als Präposition angesehen (Quelle: Wielki Stownik Jezyka Polskiego, https://wsjp.pl/index. php?id_hasla=60203\&id_znaczenia=5166959\&l=4\&ind=0, Zugang: 04.03.2021). 
wird im Deutschen dagegen als untypisch angesehen, da die zweite Komponente der gefundenen Beispiele nur vom Verb laufen abgeleitet wird. Die Beispiele hier sind gegenläufig oder beiläufig. Auch auf der polnischen Seite kommt dieser Typ selten vor und wird von solchen Wörtern wie nadprzyrodzony veranschaulicht.

Eine Gruppe, die in beiden Sprachen vorhanden ist, stellt dagegen die $\mathrm{Zu}-$ sammensetzung aus Pronomen und Adjektiv dar. Sie können in mehrere Typen eingeteilt werden. Dabei muss bemerkt werden, dass im Polnischen mehr Typen nachweisbar sind als im Deutschen. Im Polnischen sind Verbindungen wie $\mathrm{PR}+\mathrm{A}(\mathrm{N})$ und $\mathrm{PR}+\mathrm{A}(\mathrm{V})$ möglich. Der Typ PP+A (V) kommt im Polnischen anhand des Wörterbuchvergleichs nicht vor. Im Deutschen ist PU+A (N) die größte Untergruppe; im Polnischen dagegen $-\mathrm{PR}+\mathrm{A}(\mathrm{V})$. Andere Typen spielen keine bedeutende Rolle.

Tab. 7: Typen der Zusammensetzung aus Pronomen und Adjektiv

\begin{tabular}{|l|l|c|c|}
\hline \multicolumn{1}{|c|}{ Art der Zusammensetzung } & Abkürzung & Deutsch & Polnisch \\
\hline Determinativpronomen+Adjektiv (Nomen) & PD+A (N) & 3 & 1 \\
\hline Personalpronomen+Adjektiv (Verb) & PP+A (V) & 1 & 0 \\
\hline reflexives Pronomen+Adjektiv & PR+A & 1 & 0 \\
\hline reflexives Pronomen+Adjektiv (Nomen) & PR+A (N) & 3 & 1 \\
\hline reflexives Pronomen+Adjektiv (Verb) & PR+A (V) & 4 & 5 \\
\hline unbestimmtes Pronomen+Adjektiv & PU+A & 1 & 1 \\
\hline unbestimmtes Pronomen+Adjektiv (Nomen) & PU+A (N) & 10 & 1 \\
\hline unbestimmtes Pronomen+Adjektiv (Verb) & PU+A (V) & 2 & 2 \\
\hline
\end{tabular}

Der Typ PD+A (N) ist in beiden Sprachen möglich. Auf der deutschen Seite sind Beispiele wie derartig, derzeitig, diesbezüglich zu finden. Im Polnischen gilt als Beispiel hierfür das Wort tegoroczny. Solche Bildungen gelten in beiden Sprachen als idiomatisiert und unproduktiv. Darüber hinaus können sich die Adjektive im Deutschen zudem mit Personalpronomen verbinden. Das einzige Beispiel des Typs ist das idiomatisierte Wort ichbezogen. Deshalb ist das Modell unproduktiv.

Die Aufmerksamkeit sollte zugleich auf die adjektivischen Zusammensetzungen mit Reflexivpronomen gelenkt werden. Die Grundwörter der Zusammensetzungen werden im Polnischen entweder von Nomina oder von Verben abgeleitet, während im Deutschen das Adjektiv ein Simplex darstellen kann. Das einzige Pronomen ist im Deutschen das Wort selbst, und im Polnischen das entsprechende Wort sam. Als Beispiele dienen hierfür selbstbewusst, selbstgerecht $-\mathrm{PR}+\mathrm{A}$, selbstsüchtig, selbstherrlich $-\mathrm{PR}+\mathrm{A}(\mathrm{N})$,selbstvergessen, selbstklebend $-\mathrm{PR}+\mathrm{A}(\mathrm{V})$ und im Polnischen: z.B. in samoobstugowy - PR+A (N), samobójczy, samoprzylepny - PR+A (V). Zudem werden hier Zusammensetzungen mit unbestimmten Pronomina unterschieden. Als unbestimmtes Pronomen gilt unter den deutschen Beispielen meistens das Wort all, das sich mit abgeleiteten Adjektiven verbindet: 
alljährig, allmonatlich, allumfassend, allwissend. Einen Sonderfall stellt das Wort anderweit dar, in dem ander als Erstglied gilt. Im Polnischen dagegen verbinden sich die Adjektive mit dem Wort wszech-: wszechmocny - $\mathrm{PU}+\mathrm{A}$, wszechwtadny $-\mathrm{PU}+\mathrm{A}(\mathrm{N})$, wszechmogacy, wszechwiedzacy - PU+A (V). Semantisch sind hier keine Unterschiede zwischen beiden Sprachen festzustellen.

Die letzte Gruppe von zusammengesetzten Adjektiven stellen Komposita aus Verb und Adjektiv dar. Solche Bildungen treten allerdings nur im Deutschen auf und gelten nicht als untypisch. Am häufigsten sind die adjektivischen Grundwörter hierbei unabgeleitet.

Tab. 8: Typen der Zusammensetzung aus Verb und Adjektiv

\begin{tabular}{|l|l|c|c|}
\hline Art der Zusammensetzung & Abkürzung & Deutsch & Polnisch \\
\hline Verb+Adjektiv & $\mathrm{V}+\mathrm{A}$ & 91 & 0 \\
\hline Verb+Adjektiv (Nomen) & $\mathrm{V}+\mathrm{A}(\mathrm{N})$ & 15 & 0 \\
\hline Verb+Adjektiv (Verb) & $\mathrm{V}+\mathrm{A}(\mathrm{V})$ & 8 & 0 \\
\hline
\end{tabular}

Im Typ V+A können feste Muster wie -fest, -frei, -sicher oder -wert gefunden werden. Ihre Beispiele sind: bissfest, druckfest, knitterfrei, abhörsicher oder bemerkenswert und liebenswert. Andere Beispiele sind hierfür Zusammensetzungen wie: brennheiß oder denkfaul dar. Die Komposita erfüllen sowohl Spezifizierungs(schaulustig) als auch Intensivierungsfunktionen (stinknormal, sterbenskrank). Im Typ V+A (N) sind auch einige feste Muster zu sehen: -würdig (fragwürdig) oder -pflichtig (meldepflichtig). Das typische Suffix der zweiten Komponente ist -ig: schwindsüchtig, barmherzig. Im Falle des Typs V+A (V) stellen die Adjektive vor allem Partizipien dar: aufsehenerregend (Partizip I) oder gehbehindert (Partizip II). Seltener werden die zweiten Komponenten mit einem Suffix abgeleitet: baufällig. Die polnischen Wörterbuchsäquivalente von solchen Zusammensetzungen haben häufig die Form einer Ableitung (schaulustig-ciekawski; aufsehenerregend - sensacyjny, rutschfest - antypoślizgowy). Solche Komposita können wiederum als Phrasen wiedergegeben werden (lobenswert - godny pochwaty, sterbenskrank - obłożnie chory). In diesen Fällen wird die Bedeutung solcher Zusammensetzungen mit anderen Mitteln wiedergegeben.

\section{Fazit}

Angesichts der Analyse lässt sich feststellen, dass sowohl Ähnlichkeiten als auch Unterschiede in der Bildung von zusammengesetzten Adjektiven zwischen beiden Sprachen erkannt werden und obwohl in den untersuchten Wörterbüchern nicht alle möglichen Zusammensetzungen berücksichtigt wurden, sind viele Ähnlichkeiten der deutsch-polnischen kontrastiven Wortbildung auf Basis der Analyse erkennbar. 
Möglich sind im Deutschen sowie im Polnischen folgende Zusammensetzungen: Adjektiv-Adjektiv; Adverb-Adjektiv; Nomen-Adjektiv; Zahlwort-Adjektiv; Präposition-Adjektiv; Pronomen-Adjektiv. Dabei haben viele Zusammensetzungsarten eine gleiche oder ähnliche Bedeutung: Adjektiv-Adjektiv; Adverb-Adjektiv; Zahlwort-Adjektiv oder Präposition-Adjektiv. Deutlich sichtbar sind die Ähnlichkeiten in der Bildung von Kopulativkomposita. Auch etliche deutsche Determinativkomposita haben im Polnischen eine direkte formale Entsprechung, z.B.: halbnackt - pótnagi; blauäugig - niebieskooki; erstklassig - pierwszorzędny. Beide Sprachen verfügen über feste Wortbildungsmuster, die analoge Zusammensetzungen darstellen können: -ähnlich/-podobny; -sicher/-odporny. Die beschriebenen Ähnlichkeiten können als große Erleichterung im Übersetzungsprozess oder in der Fremdsprachendidaktik dienen. Diese festen Wortbildungsmuster sind auch bei Okkasionalismen anwendbar (vgl. Szkopek 2007: 274).

Andererseits dürfen die formalen Unterschiede in der Bildung von zusammengesetzten Adjektiven nicht unterschätzt werden: In erster Linie ist die Bildung der Zusammensetzungen in der deutschen Sprache viel stärker ausgeprägt als im Polnischen. Es ist vor allem darauf zurückzuführen, dass in den deutschen $\mathrm{Zu}$ sammensetzungen Komposita aus Nomen und Adjektiv eine wesentliche Gruppe darstellen, während dieser Typ im Polnischen nur mit wenigen Beispielen repräsentiert wird. Die deutschen Komposita aus Nomen und Adjektiv können jedoch in mehreren Fällen im Polnischen mit einer Adjektiv-Adjektiv-Zusammensetzung wiedergegeben werden: schneeweiß - śnieżnobialy. Darüber hinaus erscheinen im Deutschen Kombinationen, die in der zweiten Sprache nicht vorkommen, wie Verb-Adjektiv-Zusammensetzungen. Ein weiterer Unterschied besteht darin, dass die zweiten Komponenten in deutschen Komposita häufiger Simplizia darstellen, während sie im Polnischen größtenteils abgeleitet werden. Eine Art der Zusammensetzung kann allerdings mit einem anderen Zusammensetzungstyp wiedergegeben werden (z.B. Zusammensetzungen aus Nomen und Adjektiv im Deutschen als Komposita des Typs A (N)+A). Häufig stellen aber die polnischen Entsprechungen von deutschen Zusammensetzungen Ableitungen oder feste Phrasen dar.

\section{Literatur}

Donalies, Elke (2007): Basiswissen. Deutsche Wortbildung. Tübingen/Basel.

Engel, Ulrich (1999): Deutsch-polnische konstrastive Grammatik, Bd. 1-2. Heidelberg. Fleischer, Wolfgang (1982): Wortbildung der deutschen Gegenwartssprache. Tübingen.

Fleischer, Wolfgang / Barz, Irmhild (2012): Wortbildung der deutschen Gegenwartssprache. Tübingen.

Frączek, Agnieszka / Wiktorowicz, Józef (Hrsg.) (2008a): Wielki słownik niemiecko-polski. Warszawa.

Frączek, Agnieszka / Wiktorowicz, Józef (Hrsg.) (2008b): Wielki stownik polsko-niemiecki. Warszawa. 
Grzegorczykowa, Renata / Laskowski, Roman / Wróbel, Henryk (Hrsg.) (1999): Gramatyka wspótczesnego języka polskiego. Morfologia. Warszawa.

Jadacka, Hanna (2001): System stowotwórczy polszczyzny (1945-2000). Warszawa.

Szkopek, Katarzyna (2007): Wortneubildung im Text. Zu Wortbildungsmerkmalen zusammengesetzter Okkasionalismen. In. Bartosiewicz, Iwona / Szczęk, Joanna / Tworek, Artur (Hrsg.): Fundamenta Linguisticae. Wrocław/Dresden. S. 273-284.

Trost, Igor (2006): Das deutsche Adjektiv. Untersuchungen zur Semantik, Komparation, Wortbildung und Syntax. Hamburg. 